\title{
CD101, a novel echinocandin with exceptional stability properties and enhanced aqueous solubility
}

\author{
B Radha Krishnan ${ }^{1,2}$, Kenneth D James ${ }^{1,2}$, Karen Polowy ${ }^{2}$, BJ Bryant ${ }^{2}$, Anu Vaidya ${ }^{2}$, Steve Smith ${ }^{2}$ \\ and Christopher P Laudeman ${ }^{2}$
}

The echinocandins are an important class of antifungal agents. However, instability and, in some cases, lack of solubility have restricted their use to situations in which daily infusions are acceptable. CD101 is a novel echinocandin in development for topical and weekly i.v. administration that exhibits prolonged stability in plasma and aqueous solutions up to $40^{\circ} \mathrm{C}$. After incubation for $44 \mathrm{~h}$ in rat, dog, monkey and human plasma at $37^{\circ} \mathrm{C}$, the percent of $\mathrm{CD} 101$ remaining $(91 \%, 79 \%, 94 \%$ and $93 \%$, respectively) was consistently greater than that of anidulafungin $(7 \%, 15 \%, 14 \%$ and $7 \%$, respectively). Similarly, after incubation in phosphate-buffered saline at $37^{\circ} \mathrm{C}$, the CD101 remaining (96\%) was greater than that of anidulafungin $(42 \%)$. CD101 exhibited $<2 \%$ degradation after long-term storage at $40{ }^{\circ} \mathrm{C}$ as a lyophilized powder ( 9 months) and at room temperature in $5 \%$ dextrose (15 months), $0.9 \%$ saline (12 months) and sterile water (18 months). Degradation was $<7 \%$ at $40^{\circ} \mathrm{C}$ in acetate and lactate buffers (6 to 9 months at $\left.\mathrm{pH} 4.5-5.5\right)$. The chemical stability and solubility of CD101 contribute to dosing, pharmacokinetic, formulation and safety advantages over other echinocandins and should expand utility beyond daily i.v. therapy.

The Journal of Antibiotics (2017) 70, 130-135; doi:10.1038/ja.2016.89; published online 10 August 2016

\section{INTRODUCTION}

Candida is the leading cause of bloodstream infections within US hospitals and has been found to account for $22 \%$ of inpatient bloodstream infections. ${ }^{1}$ Since the introduction of caspofungin in 2001, the echinocandins have become increasingly important in the treatment of these life-threatening fungal infections. Since 2009, echinocandins have been recommended as first-line therapy against suspected or confirmed candidemia and invasive Candida infections. ${ }^{2,3}$ As potent noncompetitive inhibitors of the plasma membrane-bound $\beta-1,3$-D-glucan synthase, the echinocandins inhibit the synthesis of $\beta-1,3$-D-glucan, a structural polymer that comprises up to $60 \%$ of the cell wall of Candida spp. ${ }^{4}$ The prevalence of this polymer in certain fungi, the lack of it in mammals, the low incidence of resistance and virtually nonexistent drug interactions make the echinocandins very attractive and the safest of all existing classes of systemic antifungal agents. Despite these advantages, the pharmacokinetic and stability properties of the currently approved echinocandins impose limitations on their use. Because of short half-lives and poor oral absorption, they were each developed for once-daily administration by i.v. infusion. Their stability necessitates that the i.v. dosing preparations be used within 24 to $48 \mathrm{~h}^{5-7}$ and precludes the introduction of other dosage forms, such as topical and s.c. preparations. Consequently, the echinocandins presently are used sparingly for indications in which daily infusion of the drug is impractical. In short, the susceptibility to hydrolytic, thermal and (for micafungin) photodegradation ${ }^{7}$ of the approved echinocandins impose limitations for manufacturing, storage, usage and acceptable dosage forms for this otherwise attractive drug class.

A novel echinocandin, CD101 acetate (CD101; Figure 1), is presently being developed as a once-weekly i.v. formulation for the treatment and prevention of invasive fungal infections and also as a topical formulation for acute and recurrent vulvovaginal candidiasis. Characteristic of the echinocandins, CD101 is a cyclic hexapeptide with a lipophilic tail. It displays potency and spectrum of activity in vitro typical of the echinocandins. ${ }^{8,9}$ However, it has a distinct structural feature that confers much greater stability, leading to an exceptionally longer half-life ${ }^{10-12}$ and an improved safety profile. ${ }^{13}$ In this study, we present thermal and solution stability data for CD101 as a lyophilized powder and in various solutions, including prototype i.v. solutions. Solubility data are also presented. The stability and solubility features of CD101 not only provide advantages for manufacturing and storage, but also enable expansion of echinocandin use to include weekly i.v. infusions and topical and s.c. dosage forms.

\section{MATERIALS AND METHODS}

General

Unless otherwise indicated, all reagents were purchased from Sigma-Aldrich (St Louis, MO, USA). All solvents and modifiers were HPLC grade. HPLC analyses were performed using an Agilent 1200 Series LC with an autosampler, thermostatted column compartment and multi-wavelength detector. HPLC columns were purchased from Agilent (Santa Clara, CA, USA). Anidulafungin

${ }^{1}$ Cidara Therapeutics, San Diego, CA, USA and ${ }^{2}$ Seachaid Pharmaceuticals, Durham, NC, USA

Correspondence: Dr KD James, Seachaid Pharmaceuticals, PO Box 488, Mebane, NC 27302, USA.

E-mail: kenjames@seachaid.com

Received 20 February 2016; revised 4 May 2016; accepted 17 May 2016; published online 10 August 2016 

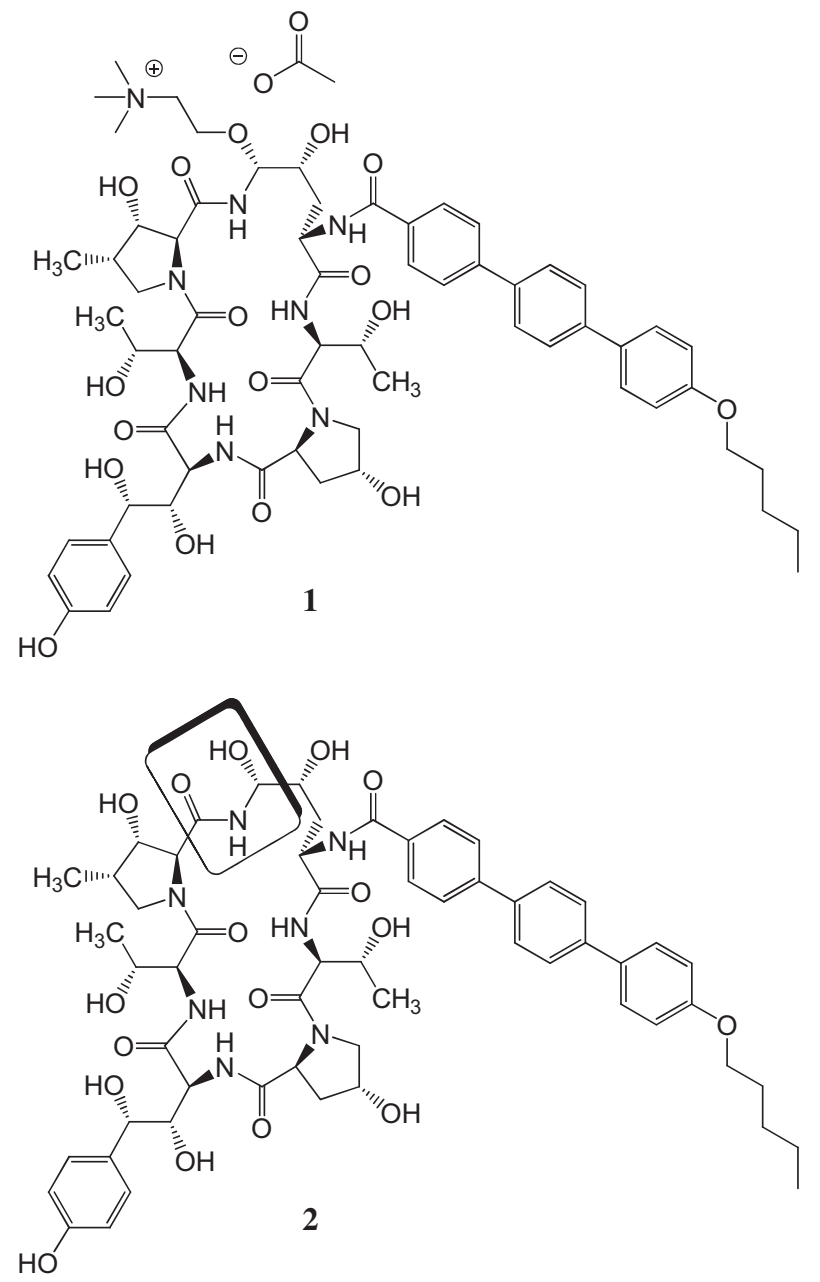

Figure 1 Structures of CD101 (1) and anidulafungin (2). The primary means of elimination in vivo for anidulafungin is chemical degradation that occurs initially at the hemiaminal region shown in the box. For CD101, the hemiaminal is replaced with a choline aminal ether that imparts greater stability and solubility to the product compound.

was obtained after isolation from commercial Eraxis (Pfizer, New York, NY, USA). The synthesis of CD101 will be described elsewhere.

\section{HPLC analyses}

All quantitative assays and purity assessments were performed using two reversed-phase HPLC methods. Method 1 was used to assess the purity of CD101 and to identify the formation of any degradation products. Method 2 was used to assess stereochemical stability at the hemiaminal ether position by separating the $\alpha$-isomer (CD101, Figure 1) from any potential $\beta$-isomer, which has the opposite stereochemistry at the site of choline ether attachment. Quantitation was performed by comparison of peak areas of test samples to the peak areas of external reference standards, analyzed in separate HPLC injections within the same set of samples. The reference standards for these studies were solutions of known concentration of previously purified and characterized lots of anidulafungin and CD101.

Method 1. An Agilent Pursuit XRs Diphenyl column (4.6 mm i.d. $\times 250 \mathrm{~mm}$ length, $3.0 \mu \mathrm{m}$ particle size) was used. Elution was performed with a column temperature of $40{ }^{\circ} \mathrm{C}$ and a gradient system with a flow rate of $1 \mathrm{ml} \mathrm{min}^{-1}$ (A: $\mathrm{H}_{2} \mathrm{O}$ with $0.1 \%$ TFA; B: acetonitrile with $0.1 \%$ TFA; \%B: $10-42$ over 5 min, $42-50$ over $20 \mathrm{~min}$ ). UV detection was performed at $300 \mathrm{~nm}$.
Method 2. An Agilent Zorbax 300SB C3 column $(4.6 \mathrm{~mm}$ i.d. $\times 150 \mathrm{~mm}$ length, $3.5 \mu \mathrm{m}$ particle size) was used. Elution was performed with a column temperature of $60^{\circ} \mathrm{C}$ and an isocratic system with a flow rate of $0.5 \mathrm{ml} \mathrm{min}{ }^{-1}$ (A: $\mathrm{H}_{2} \mathrm{O}$ with $0.3 \%$ phosphoric acid; $\mathrm{B}$ : methanol with $0.3 \%$ phosphoric acid; $50 \%$ B over $40 \mathrm{~min}$ ). UV detection was performed at $300 \mathrm{~nm}$.

\section{Stability in plasma and PBS buffer}

Stock solutions of CD101 and anidulafungin were prepared in DMSO at concentrations of $1.0 \mathrm{mg} \mathrm{ml}^{-1}$. Blank plasma (rat, dog, monkey, and human) was mixed with $10 \%$ volume of sodium phosphate buffer $(1.0 \mathrm{M}, \mathrm{pH}=7.4)$. In the control, phosphate-buffered saline (PBS, $\mathrm{pH}=7.4$ ) was used in place of plasma. Each respective matrix was mixed separately with $1.0 \%$ of the stock solutions of the test articles, bringing the starting concentration of analyte in each sample to $\sim 10 \mu \mathrm{g} \mathrm{ml}^{-1}$. The sample volume for each solution was $5.0 \mathrm{ml}$. Each stability sample was divided into aliquots of $500 \mu \mathrm{l}$ that were incubated at $37^{\circ} \mathrm{C}$. At each stability time point $(1,2,8,21$ and $44 \mathrm{~h})$, an aliquot was quenched with acetonitrile ( $500 \mu \mathrm{l}$, with internal standard) and centrifuged. The supernatant $(100 \mu \mathrm{l})$ was removed and analyzed by reversed-phase HPLC using Method 1.

\section{Stability as a lyophilized powder}

Samples from three lots of CD101 (lyophilized powder) were stored in clear borosilicate glass vials and sealed with polypropylene caps with Teflon liners at $40{ }^{\circ} \mathrm{C}$ for $6-9$ months. No stabilizers or other excipients were added to the lyophilisate. Stability was monitored over a period of 9 months using reversed-phase HPLC methods 1 and 2. Appearance was also monitored.

\section{Stability in i.v. infusion solutions}

CD101 $\left(3.33 \mathrm{mg} \mathrm{ml}^{-1}\right)$ in lactate buffer was diluted to 1.1 or $0.77 \mathrm{mg} \mathrm{ml}^{-1}$ with $5 \%$ dextrose or $0.9 \%$ saline infusion solutions, respectively. The dextrose formulation solution was stored in an infusion bag at room temperature. The saline formulation solution was stored in a sealed, clear glass vial. Both were unprotected from light for up to 15 months. No stabilizers were added to the solutions. Stability was monitored using HPLC methods 1 and 2. The assay values were reported in comparison with a reference standard and are an indication of the amount of CD101 in solution. Values between 90 and $110 \%$ of the target concentration were considered to be within the specification. Appearance was also monitored.

\section{Stability in aqueous solutions}

CD101 stock solutions (acetate buffer, lactate buffer and USP sterile water) were made at concentrations of $3.33 \mathrm{mg} \mathrm{m}^{-1}$ and were stored in clear borosilicate glass vials sealed with polypropylene caps with Teflon liners. No stabilizers or other excipients were added. The lactate and some acetate-buffered solutions were incubated at $40^{\circ} \mathrm{C}$. The sterile water and some acetate-buffered solutions were kept at room temperature and unprotected from light. Stability was monitored up to 18 months using HPLC methods 1 and 2. The assay values were reported in comparison with a reference standard. Values between 90 and $110 \%$ of the target concentration $\left(3.33 \mathrm{mg} \mathrm{ml}^{-1}\right)$ were considered to be within the specification. Appearance was also monitored.

\section{Solubility studies}

Accurately weighed CD101 (up to $25 \mathrm{mg}$ ) was dissolved in sterile water, ethanol and buffered solutions of acetate, citrate, lactate, tartrate, phosphate and Tris in clear glass vials to final volumes of $0.5 \mathrm{ml}$ at room temperature. No solubilizers or other excipients were added. Stirring was performed up to $16 \mathrm{~h}$ as necessary to form a clear solution. Any sample that contained undissolved material was centrifuged for $5 \mathrm{~min}$, and the supernatant was analyzed. The solution samples were analyzed by HPLC method 1 .

\section{RESULTS}

\section{Stability in plasma and PBS buffer}

After incubation at $37^{\circ} \mathrm{C}$ in rat, dog, monkey and human plasma, the percentage of CD101 remaining after $44 \mathrm{~h}$ was $91 \%, 79 \%, 94 \%$ and 93\%, respectively. Stability of CD101 in PBS buffer was similar 


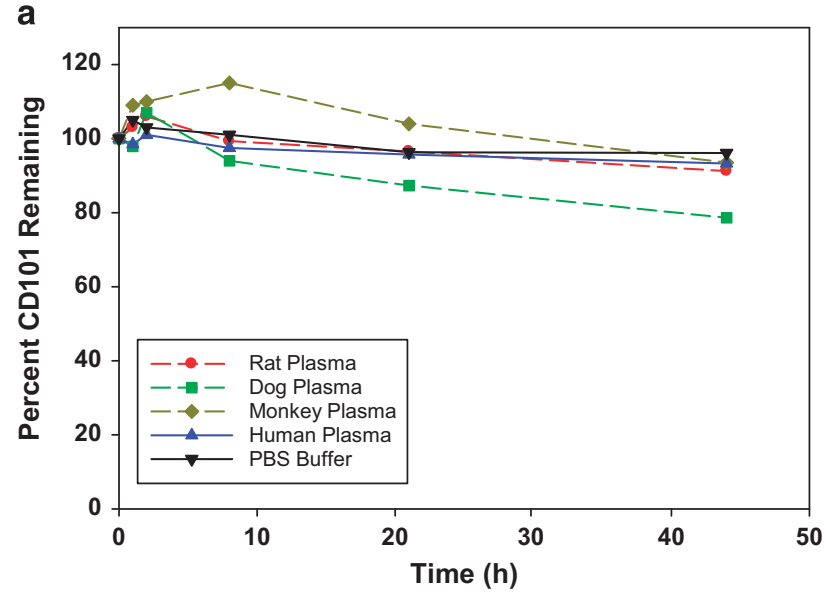

b

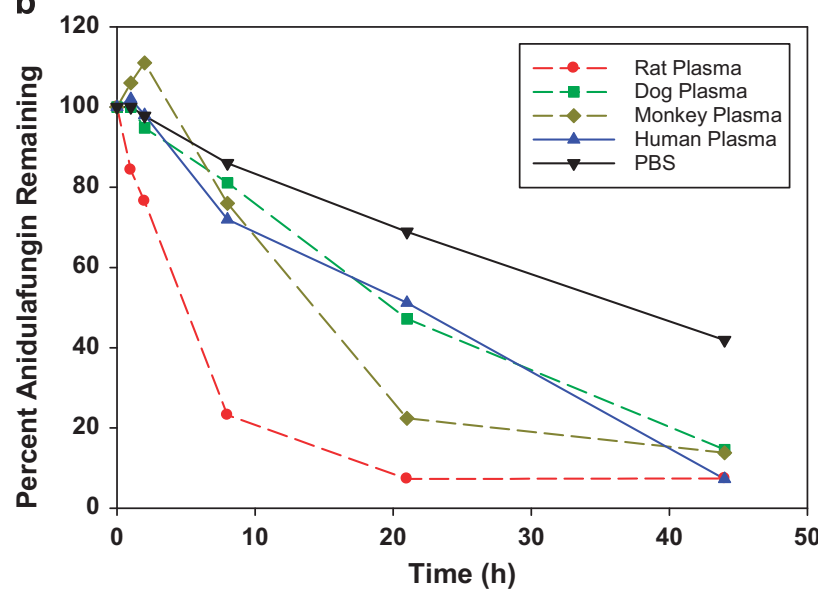

Figure 2 Degradation of CD101 (a) and anidulafungin (b) in plasma from different species and in phosphate-buffered saline (PBS) buffer. Each echinocandin $\left(10 \mu \mathrm{g} \mathrm{ml}^{-1}\right)$ was incubated at $37^{\circ} \mathrm{C}$ for $44 \mathrm{~h}$ in plasma (rat, dog, monkey and human) and PBS buffer. Reactions were assayed at various time points $(0,1,2,8,21$ and $44 \mathrm{~h})$ for the amount of intact echinocandin remaining, expressed as a percentage of the $\mathrm{Oh}$ time point concentration. No stabilizers were used in the reactions. In contrast to anidulafungin, CD101 showed very little degradation in the matrices that were tested.

(96\% remaining after $44 \mathrm{~h}$ ). The time curves for CD101 in the various matrices are shown in Figure 2a. Degradation of anidulafungin was comparatively much faster in each of the four plasma matrices, as evidenced by nearly complete degradation of anidulafungin in $<2$ days. After incubation at $37^{\circ} \mathrm{C}$ in rat, dog, monkey and human plasma, the anidulafungin remaining after $44 \mathrm{~h}$ was $7 \%, 15 \%, 14 \%$ and $7 \%$, respectively. These results were in good agreement with an earlier report demonstrating rapid chemical degradation of anidulafungin in human plasma. ${ }^{14}$ They are also consistent with a daily dosing regimen as described in the prescribing information. ${ }^{5}$ Degradation of anidulafungin was also extensive in PBS buffer ( $42 \%$ remaining after $44 \mathrm{~h}$ ), although not as rapid as in the plasma experiments. The time curves for anidulafungin in the various matrices are shown in Figure 2b.

\section{Stability as a lyophilized powder}

The results from stability studies under accelerated conditions of two lots of CD101 lyophilisates are presented in Table 1. Values reported for individual lots are the percent area under the curve for CD101 and
Table 1 Stability data of CD101 as a lyophilized powder at $40{ }^{\circ} \mathrm{C}$

\begin{tabular}{llrrrrr} 
& & \multicolumn{5}{c}{ Time (months) } \\
\cline { 3 - 7 } CD101 lot & Test & 0 & 1 & 3 & 6 & 9 \\
\hline \multirow{nnyyyyy}{*}{} & Chromatographic purity (\% AUC) & 99.0 & 98.6 & 98.4 & 98.3 & 96.5 \\
1 & $\begin{array}{l}\text { Impurity and degradation products } \\
\text { (\% AUC) }\end{array}$ & 1.0 & 1.4 & 1.6 & 1.7 & 3.5 \\
& Chromatographic purity (\% AUC) & 97.5 & 97.1 & 96.8 & 96.3 & 96.4 \\
2 & Impurity and degradation products & 2.5 & 2.2 & 3.2 & 3.3 & 3.5 \\
2 & (\% AUC) & & & & & \\
Average & Chromatographic purity (\% AUC) & 98.3 & 97.9 & 97.6 & 97.3 & 96.5 \\
Average & Impurity and degradation products & 1.8 & 1.8 & 2.4 & 2.5 & 3.5 \\
& (\% AUC) & & & & & \\
Average & \% Degradation & 0 & 0.0 & 0.6 & 0.7 & 1.7 \\
\hline
\end{tabular}

Abbreviation: AUC, area under the curve.

for the total of all impurities and degradation products. Averages for purity, impurity and degradation products and percent degradation are also presented. After storage at $40{ }^{\circ} \mathrm{C}$ for 9 months in the absence of stabilizers, CD101 evidenced only a slight increase $(\sim 1.7 \%)$ in degradation products. The average chromatographic purity after 9 months was $96.5 \%$. The emergence of a new peak indicating epimerization of the hemiaminal ether was not observed, suggesting no conversion from the $\alpha$-isomer to $\beta$-isomer.

\section{Stability of CD101 in i.v. infusion solutions}

The results from stability studies of CD101 in two different i.v. infusion solutions (5\% dextrose and $0.9 \%$ saline) are presented in Table 2. Purities are reported as the percent area under the curve for CD101 out of the total chromatogram. After storage at room temperature and exposure to light in the absence of stabilizers, CD101 exhibited no decrease in assay of active pharmaceutical ingredient in either the 5\% dextrose solution after 15 months or in $0.9 \%$ saline solution after 12 months. Degradation was minimal in each solution over the course of the study. In addition, the emergence of a new peak indicating epimerization of the hemiaminal ether was not observed, suggesting no conversion from the $\alpha$-isomer to $\beta$-isomer.

\section{Stability of CD101 in aqueous solutions}

The results from stability studies of CD101 in different aqueous solutions at varying $\mathrm{pH}$ in the absence of stabilizers are presented in Table 3. Purities are reported as the percent area under the curve for CD101 out of the total chromatogram. Degradation was slow under accelerated conditions $\left(40^{\circ} \mathrm{C}\right)$ over a period of 6 to 9 months in slightly acidic buffered solutions. CD101 displayed $<7 \%$ and $4 \%$ degradation in acetate buffers at $\mathrm{pH} 4.5$ and 6.0, respectively. Degradation was $<5 \%$ in lactate buffers ( $\mathrm{pH} 4.5$ and 5.5) over periods of 6 and 9 months, respectively, at $40^{\circ} \mathrm{C}$. In contrast, degradation was extremely low at room temperature in acetate buffers ( $\mathrm{pH} 4.5$ to 6.0 ) and sterile water over 18 months. No epimerization of the hemiaminal ether was observed in any of the samples.

\section{Solubility}

The solubility of CD101 was evaluated in various aqueous solutions, buffered solutions ( $\mathrm{pH}$ range from 4.5 to 9.5 ) and nonaqueous media. Anidulafungin was also evaluated under many of the same conditions. The solubility results are displayed in Table 4 . CD101 showed excellent solubility $\left(>150 \mathrm{mg} \mathrm{ml}^{-1}\right.$ ) in sterile water, in stark contrast to the $<0.1 \mathrm{mg} \mathrm{ml}^{-1}$ demonstrated by anidulafungin in the absence of 
Table 2 Stability data of CD101 in i.v. infusion solutions

\begin{tabular}{|c|c|c|c|c|c|c|c|c|c|}
\hline \multirow[b]{2}{*}{ CD101 infusion solution } & \multirow[b]{2}{*}{ Concentration $\left(\mathrm{mg} \mathrm{m}^{-1}\right.$ ) } & \multirow[b]{2}{*}{ Test } & \multicolumn{7}{|c|}{ Time (months) } \\
\hline & & & 0 & 1 & 3 & 6 & 9 & 12 & 15 \\
\hline \multirow[t]{2}{*}{$5 \%$ Dextrose } & 1.1 & Assay (mg ml-1) & 1.11 & 1.12 & 1.10 & - & 1.13 & 1.12 & 1.11 \\
\hline & & Purity (\% AUC) & 97.3 & 97.1 & 97.2 & - & 98.1 & 96.9 & 97.7 \\
\hline \multirow[t]{2}{*}{ 0.9\% Saline } & 0.77 & Assay (mg ml-1) & 0.80 & 0.81 & 0.79 & 0.82 & 0.83 & 0.79 & - \\
\hline & & Purity (\% AUC) & 93.7 & 93.5 & 93.7 & 94.4 & 94.2 & 94.0 & - \\
\hline
\end{tabular}

Abbreviation: AUC, area under the curve.

Table 3 Stability data of CD101 in aqueous solutions at varying $\mathrm{pH}$

\begin{tabular}{|c|c|c|c|c|c|c|c|c|c|c|}
\hline \multirow[b]{2}{*}{ CD101 solution } & \multirow[b]{2}{*}{ Conc. $\left(m g ~ m l^{-1}\right)$} & \multirow[b]{2}{*}{ Temp $\left({ }^{\circ} \mathrm{C}\right)$} & \multirow[b]{2}{*}{ Test } & \multicolumn{7}{|c|}{ Time (months) } \\
\hline & & & & 0 & 1 & 3 & 6 & 9 & 12 & 18 \\
\hline \multirow[t]{2}{*}{ Acetate buffer (20 mm, pH 4.5) } & 3.3 & RT & Assay (mg ml-1) & 3.25 & 3.26 & 3.16 & 3.32 & 3.62 & 3.70 & 3.60 \\
\hline & & & Purity (\% AUC) & 97.7 & 96.6 & 96.6 & 96.9 & 97.4 & 97.3 & 97.4 \\
\hline \multirow[t]{2}{*}{ Acetate buffer (20 mm, pH 4.5) } & 3.33 & 40 & Assay (mg ml-1) & 3.28 & 3.24 & 3.14 & 3.25 & - & - & - \\
\hline & & & Purity (\% AUC) & 97.7 & 96.0 & 94.5 & 91.2 & - & - & - \\
\hline \multirow[t]{2}{*}{ Lactate buffer (20 mm, pH 4.5) } & 3.33 & 40 & Assay (mg ml-1) & 3.18 & 3.21 & 3.11 & 3.20 & 3.16 & - & - \\
\hline & & & Purity (\% AUC) & 93.9 & 92.6 & 92.3 & 91.1 & 89.3 & - & - \\
\hline \multirow[t]{2}{*}{ Lactate buffer (20 mm, pH 5.5) } & 3.33 & 40 & Assay (mg ml-1) & 3.32 & 3.42 & 3.21 & 3.41 & 3.18 & - & - \\
\hline & & & Purity (\% AUC) & 95.6 & 93.5 & 92.2 & 92.3 & 90.9 & - & - \\
\hline \multirow[t]{2}{*}{ Acetate buffer (20 mm, pH 6.0) } & 3.33 & RT & Assay (mg ml-1) & 3.21 & 3.25 & 3.15 & 3.20 & 3.50 & 3.50 & 3.37 \\
\hline & & & Purity (\% AUC) & 97.4 & 96.7 & 96.6 & 96.0 & 97.6 & 97.5 & 97.6 \\
\hline \multirow[t]{2}{*}{ Acetate buffer (20 mm, pH 6.0) } & 3.33 & 40 & Assay (mg ml-1) & 3.24 & 3.19 & 3.10 & 3.00 & - & - & - \\
\hline & & & Purity (\% AUC) & 97.4 & 95.9 & 95.6 & 93.8 & - & - & - \\
\hline \multirow[t]{2}{*}{ Sterile water } & 3.33 & RT & Assay (mg ml-1) & 3.20 & 3.20 & 3.14 & 3.20 & 3.49 & 3.32 & 3.33 \\
\hline & & & Purity (\% AUC) & 97.3 & 96.6 & 96.7 & 96.1 & 96.7 & 97.4 & 97.4 \\
\hline
\end{tabular}

Abbreviations: AUC, area under the curve; RT, room temperature.

solubilizers. CD101 also demonstrated high solubility $\left(>45 \mathrm{mg} \mathrm{ml}^{-1}\right.$ ) in buffers made from acetate ( $\mathrm{pH} 4.5$ and 6.0), lactate ( $\mathrm{pH}$ 6.0, 6.5, 7.4 and 8.5) and Tris ( $\mathrm{pH} 7.4$ and 8.5). Solubilities for CD101 were noticeably lower in phosphate and citrate buffers in comparison with the other media evaluated. Absent solubilizers, anidulafungin did not show solubility of $>0.1 \mathrm{mg} \mathrm{ml}^{-1}$ in any of the aqueous conditions tested. It did, however, show good solubility $\left(>17 \mathrm{mg} \mathrm{ml}^{-1}\right)$ in ethanol, exceeding that observed for CD101 $\left(14 \mathrm{mg} \mathrm{ml}^{-1}\right)$.

\section{DISCUSSION}

\section{Stability in plasma}

Chemical degradation marked by cleavage of the cyclic echinocandin core is common to the currently approved echinocandins and occurs in both plasma and buffered solutions, resulting in inactive degradants that are subject to further reaction and degradation. ${ }^{15-17}$ This pathway is a main mechanism of clearance in vivo for each of these therapeutics. ${ }^{14}$ In great contrast, CD101 is highly stable in plasma from various species and in PBS buffer. As illustrated in Figure 3, the ring-opening step that initiates the chemical degradation cascade of anidulafungin and other echinocandins is disfavored in CD101 because of the presence of the hemiaminal ether moiety. Whereas the hemiaminal hydroxyl of anidulafungin leads to a highly reactive, open-chain aldehyde, the corresponding step with the hemiaminal ether of CD101 would result in an oxonium ion, which is disfavored energetically. This explanation is further supported by prior work from Damle et al. ${ }^{14}$ regarding the stability of anidulafungin in vitro and in vivo. They found that anidulafungin undergoes rapid chemical degradation in human plasma and PBS at $37^{\circ} \mathrm{C}$. The primary degradation product was itself reactive, undergoing further transformation. During in vivo studies utilizing a radioactive tag, they found that degradation products of anidulafungin persist in the body until eventual elimination in the feces. In our studies, formation of such a degradation product was not observed with CD101. We used anidulafungin as the comparator in these studies not only because of the structural similarity to CD101, but also because anidulafungin has the longest plasma half-life of the approved echinocandins across species. ${ }^{18-22}$ The unique chemical stability of CD101 has largely circumvented the main route of elimination (chemical degradation via ring opening) that is observed for anidulafungin and is likely a key contributor to the improved pharmacokinetics of CD101, although this alone may not entirely explain the long half-life that is observed in vivo across species (typically 3 -fold to 5-fold longer than that of anidulafungin). ${ }^{10}$ In addition, the lack of degradation and the absence of open-chain aldehyde formation suggest that CD101 may offer higher margins of safety with fewer dose-limiting toxicities over echinocandins that generate such reactive degradants. ${ }^{13}$

\section{Stability as a lyophilized powder}

The stability of the CD101 lyophilisate, even at $40{ }^{\circ} \mathrm{C}$ in the absence of stabilizers (Table 1), is beneficial both for manufacturing of the active pharmaceutical ingredient and for enabling formulation of a stable drug product. In manufacturing, concerns of thermal stability between purification and formulation to the drug product are abated. It is also suggestive of a potentially long shelf-life without the need of 
Table 4 Solubilities at room temperature for CD101 and anidulafungin in various solvent conditions

\begin{tabular}{|c|c|c|}
\hline \multirow[b]{2}{*}{ Solvent } & \multicolumn{2}{|c|}{ Concentration ( $\mathrm{mg} \mathrm{m}^{-1}$ ) } \\
\hline & Anidulafungin & CD101 \\
\hline Sterile water & $<0.1$ & $>150$ \\
\hline $10 \mathrm{~mm}$ acetate buffer, $\mathrm{pH} 4.5$ & ND & $>45$ \\
\hline $100 \mathrm{~mm}$ sodium acetate buffer, $\mathrm{pH} 6.0$ & ND & $>48$ \\
\hline 10 mm citrate buffer, $\mathrm{pH} 6.0$ & ND & $\sim 2$ \\
\hline 100 mm citrate buffer, pH 6.0 & ND & $\sim 0.005-0.01$ \\
\hline 100 mм tartrate buffer, pH 6.0 & ND & $\sim 0.018$ \\
\hline 100 mм lactate buffer, pH 6.0 & ND & $>47$ \\
\hline 100 mм lactate buffer, pH 6.5 & ND & $>45$ \\
\hline 10 mm phosphate buffer, $\mathrm{pH} 6.5$ & $\sim 0.001$ & $>29$ \\
\hline 100 mм phosphate buffer, pH 6.5 & $\sim 0.001$ & $\sim 0.5$ \\
\hline 10 mm phosphate buffer, pH 7.0 & $\sim 0.004$ & $>20$ \\
\hline 100 mм phosphate buffer, pH 7.0 & $\sim 0.001$ & $\sim 0.006$ \\
\hline 10 mм phosphate buffer, pH 7.4 & $\sim 0.004$ & $\sim 5$ \\
\hline 100 mm phosphate buffer, 7.4 & $\sim 0.001$ & $\sim 0.006$ \\
\hline 100 mm Tris buffer, pH 7.4 & ND & $>47$ \\
\hline 100 mm lactate buffer, pH 7.4 & ND & $>51$ \\
\hline 100 mм Tris buffer, $\mathrm{pH} 8.5$ & ND & $>47$ \\
\hline 100 mm lactate buffer, pH 8.5 & ND & $>52$ \\
\hline 10 mм phosphate buffer, pH 9.5 & 0.015 & $\sim 7$ \\
\hline 100 mм phosphate buffer, pH 9.5 & $\sim 0.002$ & $\sim 0.008$ \\
\hline Saline $(0.9 \%)$ & $\sim 0.001$ & $\sim 0.18$ \\
\hline Saline $(0.09 \%)$ & $\sim 0.005$ & $>1.4$ \\
\hline Ethanol & $>17$ & 14 \\
\hline Glycerol & 1.5 & $>23$ \\
\hline
\end{tabular}

Abbreviation: ND, not determined.

refrigeration and the minimization of loss because of short expiration dates; further studies to investigate this are underway. The increased stability of the active pharmaceutical ingredient without stabilizers has allowed development of a lyophilized formulation that is stable at room temperature and opens the door to other formulations, such as topical $^{23}$ and s.c., ${ }^{24}$ that are not viable with the currently approved echinocandins because of their insufficient stability.

\section{Stability in i.v. infusion solutions}

The stability of CD101 in infusion solutions was measured by assay and impurity profile. The former quantitatively measures the amount of CD101 remaining in solution over the course of the study. The latter provides information on levels of individual degradants arising via epimerization, hydrolysis or other mechanisms. As both the assay and the purity of CD101 remain unchanged over the course of the studies (Table 2), the results indicate that neither precipitation nor degradation occurred in the infusion bags up to 15 months in $5 \%$ dextrose or 12 months in $0.9 \%$ saline. These observations suggest that, unlike the currently approved echinocandins, infusion solutions of CD101 could be prepared without stabilizers and stored without concern of precipitation, degradation or photolysis.

\section{Stability in aqueous solutions}

CD101 showed minimal degradation at room temperature in water or acetate buffers ( $\mathrm{pH} 4.5$ and 6.0 , Table 3) At $40{ }^{\circ} \mathrm{C}$, degradation is noticeable by 1 month, becoming more evident at each time point thereafter. In general, drug was lost not to precipitation in these studies, but to formation of impurities at the higher temperatures. The
Anidulafungin

CD101
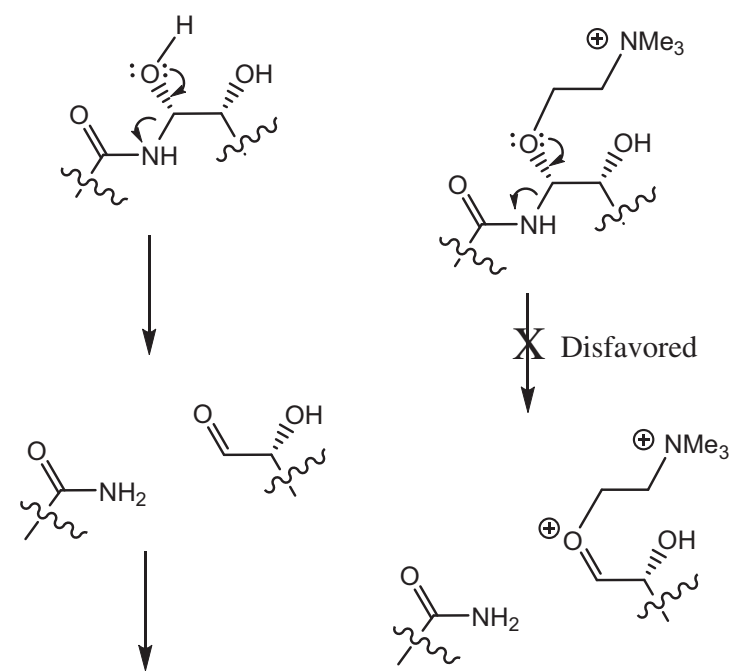

a. Further degradation
b. Intramolecular capture
c. Intermolecular capture

Figure 3 Proposed mechanism of the first step of the chemical degradation pathway for echinocandins at physiologic $\mathrm{pH}$. The cyclic anidulafungin can undergo a ring-opening event in which the hemiaminal cleaves to form a linear peptide having a terminal amide and an aldehyde from the C5 hydroxyl of the ornithine residue. This open-chain degradant is then subject to further degradation. In addition, the reactive aldehyde that is generated is then available for covalent reaction intra- or intermolecularly. This pathway is disfavored for CD101, as the first step would result in an oxonium ion. This oxonium formation is likely further discouraged by the proximity of the quaternary ammonium cation of the choline.

results from acetate buffers ( $\mathrm{pH} 4.5$ and 6.0 ) at $40{ }^{\circ} \mathrm{C}$ suggest that lower $\mathrm{pH}$ may also accelerate the degradation of $\mathrm{CD} 101$, but more studies are needed to confirm this.

\section{Solubility}

In the absence of any solubilizers, CD101 showed exceptional solubility in sterile water. Although the solubility remained high in the presence of many buffering agents representing a broad $\mathrm{pH}$ range, the solubility was greatly diminished in some buffers. The monovalent carboxylic acid buffers (acetate and lactate) were good media for $\mathrm{CD} 101$, with solubility remaining high throughout the $\mathrm{pH}$ range and buffer concentrations tested. Similarly, the solubility was also high in the amine-based Tris buffer. These observations are in contrast to the diminished solubility in the polyvalent buffers (citrate and phosphate). The effect appears to be a function of the polyvalent acid component of the buffers rather than $\mathrm{pH}$. At $\mathrm{pH} 7.4$ and under the same buffer concentrations, CD101 solubility was very high in both Tris and lactate buffers, but very low in phosphate buffer. A similar observation was observed at $\mathrm{pH}$ 6.0, at which solubility was high in acetate and lactate buffers, but very low in citrate and phosphate buffers. The influence of the polyvalent acids on solubility is further supported by the consistent observation of higher solubility at lower concentrations of polyvalent acid buffers irrespective of $\mathrm{pH}$.

The reason for the diminished solubility in the presence of polyvalent acidic buffers may be a result of ion exchange of the acetate counterion with the polyvalent acid and the potential for aggregation, but that is unclear at this time. Anidulafungin was 
selected as the comparator because of the structural similarity to CD101. The choline substitution, while having negligible effect on the activity in vitro, ${ }^{7-8}$ made a profound effect on the solubility and stability properties of this echinocandin.

\section{Conclusions}

CD101 is a novel echinocandin with a choline moiety at the C5 ornithine residue of the cyclic echinocandin core. The structural modification affords an echinocandin with increased solubility and exceptional stability in plasma, in aqueous and buffered solutions, and as a lyophilized powder. The exceptional stability in plasma and lack of degradation products likely contribute to the long half-lives across species and increased safety observed for CD101. The stability and solubility properties of CD101 suggest benefits to manufacturing and storage as well as flexibility regarding i.v. preparation in pharmacies. These properties may also enable dosage forms new to echinocandins, such as topical, s.c. and fast i.v. push preparations.

\section{CONFLICT OF INTEREST}

KDJ, BRK and CPL have been consultants for and are stockholders of Cidara Therapeutics, the company developing CD101. AV is a stockholder of Cidara Therapeutics.

\section{ACKNOWLEDGEMENTS}

This work was funded by Seachaid Pharmaceuticals. We thank David Song for contributions to the plasma study. We thank Jim Balkovec and David Hughes for helpful discussions regarding the manuscript.

1 Magill, S. S. et al. Multistate point-prevalence survey of health care-associated infections. N. Eng. J. Med. 370, 1198-1208 (2014).

2 Pappas, P. G. et al. Clinical practice guidelines for the management of candidiasis: 2009 update by the Infectious Diseases Society of America. Clin. Infect. Dis. 48, 503-535 (2009).

3 Pappas, P. G. et al. Clinical practice guideline for the management of candidiasis: 2016 update by the Infectious Diseases Society of America. Clin. Infect. Dis. 62 e1-50 (2015).

4 Bowman, S. M. \& Free, S. J. The structure and synthesis of the fungal cell wall. Bioessays 28, 799-808 (2006).

5 Package Insert, Eraxis (2012).

6 Package Insert, Cancidas (2014).

7 Package Insert, Mycamine (2013).

8 Castanheira, M., Messer, S. A., Rhomberg, P. R., Jones, R. N. \& Pfaller, M. A. Activity of a novel echinocandin biafungin (CD101) tested against most common Candida and Aspergillus species, including echinocandin- and azole-resistant strains. Abstracts, 54th Interscience Conference of Antimicrobial Agents and Chemotherapy, Washington, D C, American Society for Microbiology. M-1082 (2014).

9 Castanheira, M., Messer, S. A., Rhomberg, P. R., Dietrich, R. R. \& Pfaller, M. A. Activity of a long-acting echinocandin (CD101) and comparator antifungal agents tested against contemporary invasive fungal isolates. Abstracts, 55th Interscience Conference of Antimicrobial Agents and Chemotherapy, Washington, D C, American Society for Microbiology. M-849 (2015).

10 James, K. D., Krishnan, R., Laudeman, C. P., Polowy, K. \& Vaidya, A. Biafungin (CD101), a novel echinocandin, displays an uncommonly long half-life in multiple species. Abstracts, 54th Interscience Conference of Antimicrobial Agents and Chemotherapy, Washington, DC, American Society for Microbiology. A-693 (2014).

11 James, K. D. et al. Biafungin (CD101), a novel echinocandin, displays a long half-life in the chimpanzee, suggesting a once-weekly IV dosing option. Abstracts, 54th Interscience Conference of Antimicrobial Agents and Chemotherapy, Washington, DC, American Society for Microbiology. A-694 (2014).

12 James, K., Laudeman, C., Malkar, N., Krishnan, R. \& Polowy, K. Structure-activity relationship of a series of echinocandins and the discovery of CD101, a highly stable and soluble, once-weekly novel echinocandin. Abstracts, 55th Interscience Conference of Antimicrobial Agents and Chemotherapy, American Society for Microbiology. F-750 (2015).

13 Ong, V. et al. Preclinical evaluation shows CD101, a novel echinocandin, is highly stable with no hepatotoxicity in rats. Abstracts, 55th Interscience Conference of Antimicrobial Agents and Chemotherapy, San Diego, CA, American Society for Microbiology. A-015 (2015).

14 Damle, B. D. et al. In vitro and in vivo studies to characterize the clearance mechanism and potential cytochrome P450 interactions of anidulafungin. Antimicrob. Agents Ch 53, 1149-1156 (2009).

15 Norris, T. et al. Commercialization and late-stage development of a semisynthetic antifungal API: anidulafungin/D-fructose (Eraxis). Org. Process Res. Dev. 12 447-455 (2008).

16 Balani, S. K. et al. Metabolites of caspofungin acetate, a potent antifungal agent, in human plasma and urine. Drug Metab. Dispos. 28, 1274-1278 (2000).

17 Cross, S. A. \& Scott, L. J. Micafungin. A review of its use in adults for the treatment of invasive and oesophageal candidiasis, and as prophylaxis against Candida infections. Drugs 68, 2225-2255 (2008).

18 Kofla, G. \& Ruhnke, M. Pharmacology and metabolism of anidulafungin, caspofungin and micafungin in the treatment of invasive candidosis - review of the literature. Eur. J. Med. Res. 16, 159-166 (2011).

19 Wagner, C., Graninger, W., Presterl, E. \& Joukhadar, C. The echinocandins: comparison of their pharmacokinetics, pharmacodynamics and clinical applications. Pharmacology 78, 161-177 (2006).

$20 \mathrm{Hajdu}, \mathrm{R}$. et al. Preliminary animal pharmacokinetics of the parenteral antifungal agent MK-0991 (L-743,872). Antimicrob. Agents Ch. 41 2339-2344 (1997).

21 Niwa, T. et al. Tissue distribution after intravenous dosing of micafungin, an antifungal drug, to rats. Biol. Pharm. Bull. 27, 1154-1156 (2004).

22 Damle, B., Stogniew, M. \& Dowell, J. Pharmacokinetics and tissue distribution of anidulafungin in rats. Antimicrob. Agents Ch. 52, 2673-2676 (2008).

23 Sobel, J. D., Boikov, D., Bartizal, K. \& James, K. Evaluation of CD101 with echinocandin and azole comparators against Candida spp. isolated from patients with vulvovaginal candidiasis (VVC)Abstracts, 55th Interscience Conference of Antimicrobial Agents and Chemotherapy, San Diego, CA, American Society for Microbiology. F-752 (2015).

24 James, K. D., Krishnan, R., Polowy, K. \& Laudeman, C. P. Single-dose pharmacokinetics of biafungin (CD101), a novel echinocandin, after subcutaneous administration in rat and monkey. Abstracts, 54th Interscience Conference of Antimicrobial Agents and Chemotherapy, Washington, DC, American Society for Microbiology. POA-005 (2014).

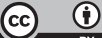

This work is licensed under a Creative Commons Attribution 4.0 International License. The images or other third party material in this article are included in the article's Creative Commons license, unless indicated otherwise in the credit line; if the material is not included under the Creative Commons license, users will need to obtain permission from the license holder to reproduce the material. To view a copy of this license, visit http:// creativecommons.org/licenses/by/4.0/

(C) The Author(s) 2017 